\title{
Unextendible Sequences in Finite Abelian Groups
}

\author{
Jujuan Zhuang \\ Department of Mathematics \\ Dalian Maritime University, Dalian, P. R. China \\ jjzhuang1979@yahoo.com.cn
}

Submitted: Oct 25, 2007; Accepted: Jun 21, 2008; Published: Jun 30, 2008

Mathematics Subject Classifications (2000): 11B75, 11P21, $11 B 50$.

\begin{abstract}
Let $G=C_{n_{1}} \oplus \ldots \oplus C_{n_{r}}$ be a finite abelian group with $r=1$ or $1<n_{1}|\ldots| n_{r}$, and let $S=\left(a_{1}, \ldots, a_{t}\right)$ be a sequence of elements in $G$. We say $S$ is an unextendible sequence if $S$ is a zero-sum free sequence and for any element $g \in G$, the sequence $S g$ is not zero-sum free any longer. Let $L(G)=\left\lceil\log _{2} n_{1}\right\rceil+\ldots+\left\lceil\log _{2} n_{r}\right\rceil$ and $d^{*}(G)=\sum_{i=1}^{r}\left(n_{i}-1\right)$, in this paper we prove, among other results, that the minimal length of an unextendible sequence in $G$ is not bigger than $L(G)$, and for any integer $k$, where $L(G) \leq k \leq d^{*}(G)$, there exists at least one unextendible sequence of length $k$.
\end{abstract}

\section{Introduction}

Let $G$ be an additively written finite abelian group, $G=C_{n_{1}} \oplus \ldots \oplus C_{n_{r}}$ its direct decomposition into cyclic groups, where $r=1$ or $1<n_{1}|\ldots| n_{r}$. Set $e_{i}=(0, \ldots, 0, \underbrace{1}_{i-t h}, 0, \ldots, 0)$ for all $i \in[1, r]$, then $\left(e_{1}, \ldots, e_{r}\right)$ is a basis of $G$. We set

$$
L(G)=\left\lceil\log _{2} n_{1}\right\rceil+\ldots+\left\lceil\log _{2} n_{r}\right\rceil,
$$

and

$$
d^{*}(G)=\sum_{i=1}^{r}\left(n_{i}-1\right) .
$$

Let $\mathcal{F}(G)$ denote the free abelian monoid over $G$ with monoid operation written multiplicatively and given by concatenation, i.e., $\mathcal{F}(G)$ consists of all multi-sets over $G$, and an element $S \in \mathcal{F}(G)$, which we refer to as a sequence, is written in the form

$$
S=\prod_{i=1}^{k} g_{i}=\prod_{g \in G} g^{v_{g}(S)},
$$


with $g_{i} \in G$, where $v_{g}(S) \in \mathbb{N}_{0}$ is the multiplicity of $g$ in $S$ and $k$ is the length of $S$, denoted by $|S|=k$. A sequence $T$ is a subsequence of $S$ if $v_{g}(T) \leq v_{g}(S)$ for every $g \in G$, denoted by $T \mid S$, and $S T^{-1}$ denote the sequence obtained by deleting the terms of $T$ from $S$. By $\sigma(S)$ we denote the sum of $S$, that is $\sigma(S)=\sum_{i=1}^{k} g_{i}=\sum_{g \in G} v_{g}(S) g \in G$. For every $l \in\{1, \ldots, k\}$, let $\sum_{l}(S)=\left\{g_{i_{1}}+\ldots+g_{i_{l}} \mid 1 \leq i_{1}<\ldots<i_{l} \leq k\right\}$, and $\sum(S)=\cup_{i=1}^{k} \sum_{i}(S)$.

Let $S$ be a sequence in $G$, we call $S$ a zero-sum sequence if $\sigma(S)=0$; a zero-sum free sequence if for any subsequence $W$ of $S, \sigma(W) \neq 0$.

In inverse zero-sum problems, for example, when we study the structure of the zerosum free sequences in $C_{n}$, set $S$ be a zero-sum sequence of length $D(G)-k$, where $D(G)$ is the well-known Davenport constant of $G$, that is the smallest integer $l \in \mathbb{N}$ such that every sequence $S$ over $G$ of length $|S| \geq l$ has a zero-sum subsequence. If $k=1$, then $S=g^{n-1}$; if $k=2$, then $S=g^{n-2}$ or $S=g^{n-3} \cdot(2 g)$, where $g \in S$ and $(g, n)=1$ (see [1],[3] and [2] etc.). The sequences $g^{n-1}$ and $g^{n-3} \cdot(2 g)$ have the same character as pointed out as follows.

Definition 1.1 Let $S$ be a zero-sum free sequence of elements in an abelian group $G$, we say $S$ is an unextendible sequence if for any element $g \in G$, the sequence $S g$ is not zero-sum free any longer.

In other words, $S$ is an unextendible sequence if and only if $\sum(S)=G \backslash\{0\}$. In fact, if $\sum(S)=G \backslash\{0\}$, then for any element $g \in G$, we get $0 \in \sum(S g)$ and $S$ is an unextendible sequence; conversely, if $S$ is unextendible, then for any $g \in G$ and $g \neq 0,0 \in \sum(S g)$ but $0 \notin \sum(S)$, and thus $-g \in \sum(S)$, that is $\sum(S)=G \backslash\{0\}$.

For any finite abelian group $G$, it is obvious that the maximal size of an unextendible sequence is $D(G)-1$.

Definition 1.2 For a finite abelian group $G$, we define $u(G)$ to be the minimal size of an unextendible sequence $S$ in $G$.

\section{On Unextendible Sequences}

We begin by describing $u(G)$ for cyclic group $C_{n}$ and for any finite abelian group.

For some real number $x \in \mathbb{R}$, let $\lceil x\rceil=\min \{m \in \mathbb{Z} \mid m \geq x\}$.

Lemma 2.1 Let $G$ be a finite abelian group of order $n$, then $u(G) \geq\left\lceil\log _{2} n\right\rceil$.

Proof. Let $S \in \mathcal{F}(G)$ be an unextendible sequence of length $u(G)$, then $\left|\sum(S)\right|=n-1$, and note that $S$ contains at most $2^{u(G)}-1$ nonempty subsequences, therefore we get $u(G) \geq\left\lceil\log _{2} n\right\rceil$.

Theorem 2.2 For any cyclic group $C_{n}, u\left(C_{n}\right)=L\left(C_{n}\right)=\left\lceil\log _{2} n\right\rceil$. 
Proof. By Lemma 2.1, it is sufficient to prove $u\left(C_{n}\right) \leq\left\lceil\log _{2} n\right\rceil$.

If $n=2^{m}$, where $m$ is a positive integer. Note that the sequence $S=\prod_{i=0}^{m-1} 2^{i}$ is an unextendible sequence of length $|S|=m=\log _{2} n$, since each integer which is smaller than $2^{m}$ can be expressed as the sum of some subsequence of $S$.

Now we assume $2^{t}<n<2^{t+1}$ for some positive integer $t$, consider the sequence $S=$ $\prod_{i=0}^{t-1} 2^{i} \cdot\left(n-2^{t}\right)$. Using the same method as above, it is easy to check that $\sum(S)=C_{n} \backslash\{0\}$ and thus $S$ is an unextendible sequence of length $|S|=t+1=\left\lceil\log _{2} n\right\rceil$.

Therefore $u\left(C_{n}\right) \leq\left\lceil\log _{2} n\right\rceil$, and thus $u\left(C_{n}\right)=\left\lceil\log _{2} n\right\rceil$.

Corollary 2.3 For any finite abelian group $G=C_{n_{1}} \oplus \ldots \oplus C_{n_{r}}$ with $1<n_{1}|\ldots| n_{r}$, $u(G) \leq L(G)$.

Proof. Consider the sequence

$$
S=\prod_{i=1}^{r}\left(\left(n_{i}-2^{\left\lceil\log _{2} n_{i}\right\rceil-1}\right) e_{i} \prod_{j=0}^{\left\lceil\log _{2} n_{i}\right\rceil-2} 2^{j} e_{i}\right),
$$

according to the proof of Theorem 2.2, $S$ is an unextendible sequence of length $|S|=$ $L(G)=\left\lceil\log _{2} n_{1}\right\rceil+\ldots+\left\lceil\log _{2} n_{r}\right\rceil$. Therefore $u(G) \leq|S|=L(G)$.

Now we discuss the existence of an unextendible sequence with certain length.

Theorem 2.4 Let $k$ be an integer satisfying $\left\lceil\log _{2} n\right\rceil \leq k \leq n-1$, then there exists an unextendible sequence $S \in \mathcal{F}\left(C_{n}\right)$ such that $|S|=k$.

Proof. We distinguish two cases:

Case 1. $\left\lceil\frac{n}{2}\right\rceil \leq k \leq n-1$. Consider the sequence

$$
S=1^{k-1} \cdot(n-1-(k-1)),
$$

clearly, $\sum(S)=C_{n} \backslash\{0\}$, and therefore $S$ is an unextendible sequence of length $|S|=k$.

Case 2. $\left\lceil\log _{2} n\right\rceil \leq k<\left\lceil\frac{n}{2}\right\rceil$. In this case, $n \geq 7$. Set $t=k-\left\lceil\log _{2} n\right\rceil$, then $0 \leq t<$ $\left\lceil\frac{n}{2}\right\rceil-\left\lceil\log _{2} n\right\rceil$. If $t=0$, by Theorem 2.2 we are done. Now suppose $0<t<\left\lceil\frac{n}{2}\right\rceil-\left\lceil\log _{2} n\right\rceil$, set $2^{i} \leq t<2^{i+1}$ where $i \in \mathbb{N}_{0}$, we consider the following subcases:

Subcase 1. $2^{i} \leq t<2^{i+1}$, and $i+1 \leq\left\lceil\log _{2} n\right\rceil-2$, then

$$
S=\prod_{j=0}^{\left\lceil\log _{2} n\right\rceil-2} 2^{j} \cdot 2^{-(i+1)} \cdot\left(n-2^{\left\lceil\log _{2} n\right\rceil-1}\right) \cdot 1^{t} \cdot\left(2^{i+1}-t\right)
$$

is an unextendible sequence of length $|S|=t+\left\lceil\log _{2} n\right\rceil=k$.

Subcase 2. $2^{\left\lceil\log _{2} n\right\rceil-2} \leq t<\left\lceil\frac{n}{2}\right\rceil-\left\lceil\log _{2} n\right\rceil$. If $t<n-2^{\left\lceil\log _{2} n\right\rceil-1}$, we consider

$$
S=\prod_{j=0}^{\left\lceil\log _{2} n\right\rceil-2} 2^{j} \cdot 1^{t} \cdot\left(n-2^{\left\lceil\log _{2} n\right\rceil-1}-t\right),
$$


otherwise, $t \geq n-2^{\left\lceil\log _{2} n\right\rceil-1}$. Noting that

$$
2^{\left\lceil\log _{2} n\right\rceil-2}+n-2^{\left\lceil\log _{2} n\right\rceil-1}=n-2^{\left\lceil\log _{2} n\right\rceil-2}>\left\lceil\frac{n}{2}\right\rceil-\left\lceil\log _{2} n\right\rceil>t,
$$

we take

$$
S=\prod_{j=0}^{\left\lceil\log _{2} n\right\rceil-3} 2^{j} \cdot 1^{t+1} \cdot\left(2^{\left\lceil\log _{2} n\right\rceil-2}+n-2^{\left\lceil\log _{2} n\right\rceil-1}-t-1\right) .
$$

Then $S$ is an unextendible sequence of length $|S|=t+\left\lceil\log _{2} n\right\rceil=k$. This completes the proof.

For any finite abelian group $G$, we have the following theorem.

Theorem 2.5 Let $G$ be a finite abelian group. Then for every $k \in\left[L(G), d^{*}(G)\right]$, there exists an unextendible sequence $S \in \mathcal{F}(G)$ of length $|S|=k$.

Theorem 2.5 comes from the following observation.

Lemma 2.6 Let $G=G_{1} \oplus G_{2}$, and $S_{i} \in \mathcal{F}\left(G_{i}\right)$ for $i \in\{1,2\}$. Then $S_{1} S_{2} \in \mathcal{F}(G)$ is unextendible if and only if both $S_{1}$ and $S_{2}$ are unextendible.

Proof. It is obvious.

Proof of Theorem 2.5. Let $G=C_{n_{1}} \oplus \ldots \oplus C_{n_{r}}$ be its direct decomposition into cyclic groups. Write $k$ in the form $k=k_{1}+\ldots+k_{r}$, where $\left\lceil\log _{2} n_{i}\right\rceil \leq k_{i} \leq n_{i}-1$. By Theorem 2.4, there exist unextendible sequences $S_{i} \in \mathcal{F}\left(C_{n_{i}}\right)$ of length $k_{i}$, and put $S=S_{1} \cdot \ldots \cdot S_{r}$, then by Lemma 2.6, $S \in \mathcal{F}(G)$ is an unextendible sequence of lengt $|S|=k$.

It is evident that any zero-sum free sequence $S$ in $G$ can be extended into an unextendible sequence. We can extend $S$ by choosing a series elements $g_{1}, \ldots, g_{r}$ in a natural process such that $\left|\sum\left(S g_{1}\right)\right|=\max \left\{\left|\sum(S g)\right| \mid g \in G\right\}$, and $\left|\sum\left(S g_{1} \ldots g_{i} g_{i+1}\right)\right|=$ $\max \left\{\mid \sum\left(S g_{1} \ldots g_{i} g\right) \| g \in G\right\}$ for any $i=2, \ldots, r$.

\section{Acknowledgements}

I would like to thank Professor Weidong Gao for bringing this problem to my attention. Many thanks belong to the referee for several very helpful comments and suggestions.

\section{References}

[1] J. D. Bovey, P. Erdős and I. Niven, Conditions for zero sum modulo n, Canad. Math. Bull., 18(1975), 27-29.

[2] W. D. Gao and A. Geroldinger, On Long minimal zero sequences in finite abelian groups, Periodica Math. Hungarica, 38(3)(1999), 179-211.

[3] W. D. Gao, An addition theorem for finite cyclic groups, Discrete Math., 163(1997), 257-265. 